\title{
Interaction of mindfulness disposition and instructional self- talk on motor performance: a laboratory exploration
}

\author{
Yi-Hsiang Chiu ${ }^{\text {Equal first author, } 1}{ }^{\text {, Frank J.H. Lu }}{ }^{1}$, Diane L Gill ${ }^{2}$, Tzu-Wen Lin ${ }^{3}$, Chiu-Chen Chang ${ }^{4}$, Shu-Ching Wu ${ }^{\text {Corresp. }}$ \\ Equal first author, 5 \\ 1 Department of Physical Education, Chinese Culture University, Taipei, Taiwan, Taiwan \\ 2 Department of Kinesiology, University of North Carolina at Greensboro, Greensboro, North Carolina, United States \\ 3 Department of Physical Education, National Taiwan Normal University, Taipei, Taiwan, Taiwan \\ 4 Department of Kinesiology, Health, and Leisure Studies, National University of Kaohsiung, Kaohsiung, Taiwan, Taiwan \\ 5 Center for General Education, Ling-Tung University, Taichung, Taiwan, Taiwan \\ Corresponding Author: Shu-Ching Wu \\ Email address: wusu@teamail.Itu.edu.tw \\ In considering that high mindfulness disposition individuals possess a unique ability to \\ maintain attention and awareness, and attention is one of the key mechanisms of \\ instructional self-talk, the purpose of this study was to examine the interaction of \\ mindfulness disposition and instructional self-talk on motor performance. Forty-nine \\ college students (Mage $=18.96 \pm 1.08$ ) with high/low mindfulness disposition (high $n=23$; \\ low $n=26$ ) selected out of 126 college students performed a discrete motor task (standing \\ long jump) and a continuous motor task (line tracking task) under instructional and \\ unrelated self-talk conditions. Two separate 2 (self-talk type) X 2 (high/low mindfulness) \\ mixed design ANOVA statistical analyses indicated that mindfulness disposition interacted \\ with unrelated self-talk in the line tracking task. Specifically, low mindfulness participants \\ performed poorer than high mindfulness participants in line tracking task under unrelated \\ self-talk. Further, participants performed better in both standing long jump and line \\ tracking under instructional self-talk than unrelated self-talk. Results not only revealed the \\ triangular relationships among mindfulness, self-talk, and motor performance but also \\ indirectly support the role of attention in self-talk effectiveness. Limitations, future \\ research directions, and practical implications were discussed.
}


1 Interaction of Mindfulness Disposition and Instructional Self-Talk on Motor Performance: A

2

3 Yi-Hsiang Chiu ${ }^{1}$, Frank J.H. Lu ${ }^{1}$, Diane L. Gill ${ }^{2}$, Tzu-Wen Lin ${ }^{3}$, Chiu-Chen Chang ${ }^{4}$

4

5

6

$7{ }^{1}$ Department of Physical Education, Chinese Culture University

$8{ }^{2}$ Department of Kinesiology, University of North Carolina at Greensboro

$9{ }^{3}$ Department of Physical Education, National Taiwan Normal University

10

11

12

13

14

15

16

17

18

19

20

21

22

23

24

25

26

27

28

29
\& Shu-Ching $\mathrm{Wu}^{5^{*}}$

${ }^{4}$ Department of Kinesiology, Health, and Leisure Studies, National University of Kaohsiung

${ }^{5}$ Center for General Education, Ling-Tung University
Correspondence concerning this article should be addressed to Shu-Ching $\mathrm{Wu}$ Assistant Professor, Center for General Education, Ling-Tung University, TAIWAN E-mail: wusu@teamail.ltu.edu.tw

5 Interaction of Mindfulness Disposition and Instructional Self-Talk on Motor Performance: A

6 Laboratory Exploration

\section{7}

8

\section{Abstract}


30 In considering that high mindfulness disposition individuals possess a unique ability to

31 maintain attention and awareness, and attention is one of the key mechanisms of instructional

32 self-talk, the purpose of this study was to examine the interaction of mindfulness disposition and

33 instructional self-talk on motor performance. Forty-nine college students (Mage $=18.96 \pm 1.08$ )

34 with high/low mindfulness disposition (high $n=23$; low $n=26$ ) selected out of 126 college

35 students performed a discrete motor task (standing long jump) and a continuous motor task (line

36 tracking task) under instructional and unrelated self-talk conditions. Two separate 2 (self-talk

37 type) X 2 (high/low mindfulness) mixed design ANOVA statistical analyses indicated that

38 mindfulness disposition interacted with unrelated self-talk in the line tracking task. Specifically,

39 low mindfulness participants performed poorer than high mindfulness participants in line

40 tracking task under unrelated self-talk. Further, participants performed better in both standing

41 long jump and line tracking under instructional self-talk than unrelated self-talk. Results not only

42 revealed the triangular relationships among mindfulness, self-talk, and motor performance but

43 also indirectly support the role of attention in self-talk effectiveness. Limitations, future research

44 directions, and practical implications were discussed. 
46 Self-talk is one of the most popular skills in psychological skills training (Gould, Tammen,

47 Murphy, \& May 1991, p. 121), and one that has been widely used by performers (Weinberg \&

48 Gould, 2015; Williams \& Krane, 2015). Self-talk is so widely used by athletes and exercisers

49 because research shows that self-talk can effectively enhance sport and motor performance

50 (Edwards, Tod, \& McGuigan, 2008; Kolovelonis, Goudas, \& Dermitzaki, 2011; Dickens, Van

51 Raalte, \& Hurlburt, 2018; Hardy, Comoutos, \& Hatzigeorgiadis, 2018; Hatzigeorgiadis,

52 Zourbanos, Galanis, \& Theodorakis, 2011; Weinberg, Miller, \& Horn, 2012). Also, the benefits

53 of self-talk have been further supported by both narrative and systematic reviews (e.g., Hardy,

54 2006; Hatzigeorgiadis et al., 2011; Tod, Hardy, \& Oliver, 2011). According to Hatzigeorgiadis

55 and colleagues (Hatzigeorgiadis et al., 2011), the global effect size of self-talk on motor

56 performance is positive and moderate (effect size, $\mathrm{ES}=0.48$ ) but ranges from 0.22 to 1.31. To

57 deepen our knowledge of the effects of self-talk on motor performance, Tod and colleagues (Tod

58 et al., 2011) suggested that researchers not only continue examining the "first-generation

59 questions", that is, the effects of self-talk on performance; but also explore the "second-

60 generation questions", that is, investigation of moderators and mediators underlying the self-talk-

61 motor performance relationship, such as self-efficacy (Chang, Ho, Lu et al., 2014), self-

62 confidence (Hatzigeorgiadis, Zourbanos, Mpoumpaki, \& Theodorakis, 2009), and task

63 characteristics (Hatzigeorgiadis, Zourbanos, Galanis, \& Theodorakis, 2011). Hence, the aim of 
64 this study was to explore the effects of instructional self-talk on motor performance, specifically,

65 the mechanism underlying self-talk-performance relationship.

66 Theoretically, self-talk is a cognitive strategy that individuals use to talk to themselves

67 either silently or aloud to interpret lived perceptions, to change evaluations and beliefs, and to

68 give instructions or reinforcements (Hardy, Gammage, \& Hall, 2001; Hardy, 2006). For

69 example, a tennis player may talk to himself and say "...hit the ball to the left bottom corner..."

70 or "...forget about the last point, now focus on the next point," or "common on, you can it!" All

71 these statements, either spoken loudly or silently, are good examples of self-talk. According to

72 the extant literature, self-talk includes the thoughts that a performer expresses in language or

73 statement, and uses implicitly or explicitly before, during or after the execution of an activity or a

74 sports skill (Zetou, Nikolaos, \& Evaggelos, 2014). Zetou and colleagues (Zetou et al., 2014)

75 contended that the self-talk process usually happens unconsciously; it affects not only the

76 performers' feelings but also their actions.

77 Notably, self-talk can be of different types including positive, negative, motivational, and

78 instructional self-talk. Among all types of self-talk, instructional self-talk is particularly worthy

79 of further examination because students learning a motor skill are given many verbal instructions

80 (McGill, 2003; pp.247-258). Instructional self-talk occurs when task performers have to focus on

81 technical, tactical, or kinesthetic components of motor tasks (Hardy, 2006; Hardy et al., 2001; 
82 Hatzigeorgiadis, Theodorakis, \& Zourbanos, 2004). Therefore, when performers apply

83 instructional self-talk they need to emphasize the cognitive components of motor tasks. By doing

84 so, performers can direct or redirect their attention to the task-relevant cues when performing

85 (Hardy, 2006; Hatzigeorgiadis et al., 2004; Landin, 1994; Nideffer, 1993; Theodorakis,

86 Weinberg, Natsis, Douma, \& Kazakas, 2000).

87 Empirical studies examining the effects of instructional self-talk on motor performance

88 yielded mixed results. For example, Theodorakis and colleagues (Theodorakis et al., 2000)

89 assigned 72 male soccer players to shoot at a goal from a distance of 15 yards. Results found the

90 instructional group performed better than the control group. Similar results were also found in

91 studies by using different motor tasks such as basketball passing accuracy and speed (Boroujeni

92 \& Shahbazi, 2011), softball throwing accuracy (Chang, Ho, Lu et al., 2014), handball accuracy

93 (Zourbanos, Hatzigeorgiadis, Bardas, \& Theodorakis, 2013), and basketball free throw shooting

94 accuracy (Abdoli, Hardy, Riyahi, \& Farsi, 2018). In contrast, some research found instructional

95 self-talk has no effect on the motor task. For example, Zourbanos and colleagues (Zourbanos et

96 al., 2013) assigned 40 primary school students to perform handball throwing accuracy with the

97 dominant hand and found no difference between instructional self-talk and control group. Similar

98 results were also found in Theodorakis and colleagues' (Theodorakis et al., 2000) early research

99 examining the effect of instructional self-talk on a 3-min endurance sit-up task. Recently, Hardy 
100 and colleagues (Hardy, Begley, Anthony, \& Blanchfield, 2015) compared the effects of

101 motivational self-talk and instructional self-talk on Gaelic football shooting accuracy using the

102 non-dominant foot. Results indicated there was no difference between the two types of self-talk

103 on shooting accuracy in skilled athletes.

104 The mixed results of the effects of instructional self-talk on motor performance prompted

105 researchers to propose factors underlying the relationship between instructional self-talk and

106 motor performance. Specifically, the attentional process during instructional self-talk has been

107 suggested by researchers. Theodorakis and colleagues (Theodorakis et al., 2000, p.266)

108 explained that the use of instructional self-talk involves attentional process; performers not only

109 have to focus on the relevant cues in the environment but also have to be aware of the specific

110 technical and mechanical aspects of the skills. Zourbanos and colleagues (Zourbanos et al., 2013,

111 p. 929) proposed that when performers are young and have no experience on a motor task (e.g.,

112 novel skill) the attentional demands are very high. Zourbanos and colleagues (Zourbanos et al.,

113 2013, p. 929) explained that by using instructional self-talk performers can focus on the key

114 elements of the movement and dismiss irrelevant stimuli. Thus, it seems that attention might

115 underlie the instructional self-talk and motor performance relationship.

116 Attention is "the behavioral and cognitive process of selectively concentrating on a discrete

117 aspect of information, whether deemed subjective or objective while ignoring other perceivable 
118 information" (Anderson, 2004; p. 519). Attention is also referred to as a state of effort and

119 arousal (Schmidt \& Lee, 2011), and the cognitive ability "to possess something in the mind

120 consciously or unconsciously in a clear and vivid form of one out of what seem several

121 simultaneous objects or trains of thought (Anderson, 2004; p. 519)". Motor behavior scholars

122 suggest that attention allows performers to allocate limited cognitive resources and handle it

123 effectively. By doing so, they can successfully achieve behavioral goals (Schmidt \& Lee, 2011;

124 97-99).

125 In considering the importance of attention in motor performance, we propose that

126 performers' mindfulness disposition- a behavioral tendency to be attentive- might influence the

127 relationship between instructional self-talk and motor performance. According to Brown and

128 Ryan (2003, p.822-823) mindfulness disposition refers to “...tendency for having an open or

129 receptive awareness and attention which is reflected in a more regular or sustained

130 consciousness of ongoing events and experiences." Many studies support that high mindfulness

131 individuals have high attention ability and perform better on motor tasks than low mindfulness

132 performers (Djikic, Langer, \& Stapleton, 2008; Kee, Chatzisarantis, Kong, Chow, \& Chen, 2012;

133 Kee \& Liu, 2011; Mills \& Allen, 2000). Thus, when examining the effects of instructional self-

134 talk on motor performance it is imperative to examine participants' mindfulness disposition

135 because their attention tendency might be a key variable affecting the instructional self-talk and 
136 motor performance relationship.

137 In addition, the triangular relationship among instructional self-talk, mindfulness

138 disposition, and motor performance suggests several theoretical questions that previous research

139 has never explored. First, is the role of mindfulness disposition on motor performance. Past

140 research examining the effects mindfulness on motor performance can be classified as (a) does

141 mindfulness training influence motor performance (e.g., Kee et al., 2012; 2013; Mills \& Allen,

142 2000; Zhang et al., 2016), or (b) how does mindfulness disposition influence motor performance

143 (e.g., Kee \& Liu, 2012). Generally, research has found mindfulness induction enhances motor

144 performance (e.g., Kee et al., 2012; 2013; Mills \& Allen, 2000; Zhang et al., 2016), or high

145 mindful disposition participants performed better than low mindful individuals (e.g., Kee \& Liu,

146 2012). Despite these efforts, research examining the effects of self-talk on motor performance

147 has not examined how mindfulness disposition influences different types of motor tasks.

148 According to Schmidt and Lee (2011), motor tasks can be classified as discrete, continuous,

149 or serial; and, each type of motor task requires a different attentional process. A discrete task,

150 such as basketball free throw or soccer kicking, which is characterized by a recognizable

151 beginning and end, requires performers to pay attention to external stimuli (e.g., target or goal),

152 and then to act. Generally, the movement time for discrete motor tasks is relatively short. For a

153 continuous task, such as swimming, steering a car, or tracking, which is characterized by having 
154 no recognizable beginning and end, performers must pay and maintain attention over a certain

155 period until the task is completed. By doing so, performers can complete the motor task

156 correctly. A serial motor task is neither discrete nor continuous, but usually comprised of a series

157 of individual movements tied together in time to make some discrete tasks "whole" such as

158 playing the piano or performing a gymnastics routine. It requires performers to pay and maintain

159 attention to both individual movement and the whole routine of the motor task. Thus, will those

160 with different mindful dispositions perform differently on different types of motor tasks during

161 self-talk needs further examined. Past research on the effects of instructional self-talk on motor

162 performance generally focused on one type of motor task, such as softball throwing (e.g., Chang

163 et al., 2014), soccer shooting (e.g., Thodorakis et al., 2000) or basketball passing (e.g., Boroujeni

164 \& Shahbazi, 2011), without comparing how self-talk influences different types of motor tasks.

165 Thus, will instructional self-talk has the same effects on different motor tasks is another question

166 to be examined.

167 Moreover, past research examining the self-talk and motor performance relationship

168 generally designed a control group such as "unrelated self-talk" (e.g., Chang et al., 2014) or

169 summary feedback (e.g., Theodorakis et al., 2000), to prevent participants from automatically

170 using another type of self-talk (e.g., motivational, positive, or negative) that might contaminate

171 the experimental effects. Under such experimental control, will unrelated self-talk interact with 
172 mindful disposition on different motor tasks is worthy of investigation.

173 Further, Lewin (1935) proposed an interactionist approach of personality suggests that

174 human behavior is the function of person and environment. $B=f(P, E)$.Thus, when researchers

175 examining the effects of self-talk on motor performance, they must consider that mindfulness

176 disposition might interact with self-talks on different type of motor tasks. Using the

177 aforementioned triangular relationships among mindfulness disposition, self-talk and motor tasks

178 as an example. For high mindful individuals, they may perform well on motor task that requires

179 the high ability of attention (e.g., continuous motor task) even under unrelated self-talk. In

180 contrast, for low mindfulness individuals, they might perform poorly in the motor task that

181 requires high attention under unrelated self-talk but remains unaffected for motor task that

182 requires a short time of attention (e.g., discrete motor task). Therefore, to examine the interaction

183 of mindfulness disposition and self-talk on different motor tasks can be a starting point to solve

184 the puzzle of why instructional self-talk cannot lead to positive effects for motor performance.

185 Built on the above rationale, the purpose of the present study was to examine the interactive

186 effects of mindfulness disposition and self-talk on motor performance. We proposed the

187 following hypotheses: (i) Mindfulness disposition would interact with self-talk in standing long

188 jump. (ii) Mindfulness disposition would interact with self-talk in line tracking. 


\section{Phase 1: Selection of Participants}

191 First, we recruited participants in PE classes at a university. We informed participants that

192 the purpose of the study was to explore college students' general psychological disposition and

193 motor skills. Participants who were interested in this study signed a consent form and completed

194 a demographic questionnaire and a Chinese version of the mindful attention awareness scale

195 (MAAS). The MAAS (Brown \& Ryan, 2003) consists of 15 items for assessing mindfulness

196 disposition. The items are comprised of statements about everyday experiences for gauging

197 frequency of receptive awareness of and attention to present-moment events and experiences. A

198 sample question is "... I rush through activities without being really attentive to them." The 6-

199 point scale, ranging from 1 (almost always) to 6 (almost never), was used. Higher mean scores

200 derived from all 15 items correspond to a high mindfulness disposition. Previous work suggests

201 that MAAS is a single factor scale and has acceptable validity and reliability (alpha coefficients

202 above .80, Brown \& Ryan, 2003). The alpha coefficient of the scale for the current sample was

203 90. It took about 15 minutes to complete a consent form, demographic questionnaire, and

204 MMAS.

205 A total of 126 participants voluntarily completed the questionnaire package. Based on their

206 MAAS scores, we identified 31 participants as high mindfulness (top 25\% of all the participants)

207 and 31 participants as low mindfulness (bottom 25\% of all the participants). Later, 23 
208 participants in the high mindfulness group and 26 in the low mindfulness group agreed to

209 participate in the experiment ( Mage $=18.96 \mathrm{yrs}, \mathrm{SD}=1.08$; females $=23$, males $=26$ ). Before

210 participating in the formal experiment, those 49 participants signed a consent form and

211 participated in the formal experiment. We used $\mathrm{G} *$ Power to estimate the experiment sample size.

212 With 2 by 2 mixed design and set alpha $=0.05$, the estimated sample size was 48 . Descriptive data

213 for participants are presented in Table 1.

Insert table 1 about here

\section{Phase 2: Formal Experiments}

217 To allow participants to learn self-talk skills, we developed a self-talk script with a

218 professor of sports psychology and an expert familiar with psychological skill training. Then, we

219 displayed this self-talk script on an instruction sheet before performing the assigned motor task.

220 Two types of self-talk (i.e., instructional and unrelated self-talk) were applied to perform two

221 motor tasks - standing long jump and a line tracking test. To apply instructional self-talk for the

222 line tracking test, participants had to talk to themselves the words like "focus on the center of the

223 groove of the panel and move it as fast as possible!" To apply unrelated self-talk for the line

224 tracking test, we refer to Chang and colleagues' $(2014$, p.141) method by asking participants to

225 talk to themselves with words like " the weather today, my clothes' colors, or my pets' names." 
226 To apply instructional self-talk for the standing long jump, participants had to talk to themselves

227 with words like "look at the front landing spot, swing the arms, relax, and jump forward as far as

228 possible!" To apply unrelated self-talk for the standing long jump, participants had to talk to

229 themselves with words like " the weather today, my clothes' colors, or my pets' names."

230 Self-talk manipulation check.

231 We adopted Kolovelonis and colleagues' suggestion (Kolovelonis et al., 2011) to check whether

232 participants followed our design in performing motor tasks under different self-talk

233 interventions. We used a self-talk check question for participants after each performance attempt.

234 The question was "during standing long jump/line tracking tasks did you use the self-talk from

235 self-talk sheet as is instructed?" The response options ranged from " 1 " absolutely not to " 5 "

236 completely so.

237 Motor performance measure.

$238 \quad$ Standing long jump task.

239 We adopted Westphal and Porter's (2013) suggestion by using the standing long jump to

240 test participants' discrete motor skill performance. The standing long jump was used to assess

241 participants' muscular power and strength. The task was conducted in a regular standard-sized

242 plastic pad with two lines indicating the distance in centimeters. During the test, the participants

243 were instructed to perform warm-up until ready to act (about 3-4 min). To begin with the 
244 experiment, participants had two warm-up attempts with $30 \%$ and $60 \%$ intensity of jumping.

245 Then, the participants were asked to execute six maximal attempts for official testing and asked

246 to jump as far as possible. The longest distance was recorded for further analysis.

\section{Hand-eye coordination of fine line tracking test.}

We used the Austrian made "Visual Pursuit Test of the Vienna Test System (VTS,

249 Schuhfried $\mathrm{GmbH}$ ) for the experiment. The VTS is a computerized psychological assessment tool for perceptual-motor skill testing (Schmid, Sauter, Stepansky, Lobentanz, \& Zeitlhofer, 2005). We used Motor Performance Series Workboard (MLS), one of the devices of VTS to measure participants' continuous motor skill performance. According to Fleishman's (1954) conceptualization of perceptual-motor skill tests, MLS is a very reliable and valid test for continuous motor skills. The MLS requires dynamic and static ability in coordinating fingerrequires participants to perform a single hand line tracking. The size of the work board is $300 \mathrm{X}$ $300 \times 15 \mathrm{~mm}$, and there are several holes, grooves, and contact surfaces. There are two pens on

258 the work board, one is black on the right and one is red on the left. In this study, participants

259 used their preferred hand in sitting position to perform the motor task, but their bodies cannot

260 touch any part of the work board. To begin with the test, participants pick up the electronic pen

261 and touch the starting point, then move along with the path of the groove until touching the finish 
262 point. While performing the task, the electronic pen cannot leave the upper edge of the work

263 board nor touch the side or bottom. If the pen hits the edge of the groove, it is recorded as a

264 mistake. More errors represent poorer performance in the line tracking motor skill task. We used

265 frequencies of mistakes for further analysis.

266 Experimental procedures.

267 Prior to data collection, we gained approval from the local institutional review board

268 (TSMH IRB No.18/ Protocol No.108-B). Before formal experiments, all participants were given

269 a short lecture to introduce the purpose of our study, the content of self-talk, and the procedures

270 to perform motor tasks. To avoid Hawthorne effects (Thomas, Nelson, \& Silverman, 2015), we

271 just told the participants that this is an experiment to examine the relationship between language

272 use and motor performance. What they need to do was just try their best during the experiment.

273 For formal experiments, participants experienced two self-talk conditions with counterbalanced

274 order. The participants were asked to select the self-talk condition by drawing lots. Then

275 participants completed 6 trials of standing long jump and 4 trials of line tracking test. To avoid

276 fatigue effects, we gave participants a 5-minute break after standing long jump. Also, during

277 each trial of line tracking, we gave 3 minutes of break. As stated earlier, the longest standing

278 long jump and the best line tracking performance (i.e., the fewest mistakes) were used for further

279 analysis. Before they performed the official attempt, the experimenters displayed the self-talk 
280 scripts. Participants selected one self-talk item from the script and then verbally and loudly spoke

281 the script to make sure they understand the words. After this step, the participants started to

282 perform a formal test. Before each trial, they had to speak these self-talk scripts either loudly or

283 silently before performing the motor tasks. The self-talk manipulation check was then

284 administrated to confirm the appropriate use of specific self-talk following each official attempt.

285 All these experiments were conducted in a quiet room of a sport psychology lab. When

286 experiments were conducted, no other person was present in order to reduce social facilitation

287 effects. Further, to avoid other unnecessary effects on experiments, we did not provide any

288 feedback on the performance. It took about 40 minutes for each participant to complete the

289 whole experiment.

290 Statistical analyses.

291 Two separate $2 \times 2$ (self-talk $\times$ high vs. low mindfulness disposition) mixed design

292 ANOVA were used to compare the effects of self-talk and high vs low mindfulness disposition

293 on standing long jump and line tracking task. Significant effects were followed-up with multiple

294 comparisons with Bonferroni correction and Greenhouse Geisser correction in order to meet the

295 sphericity assumption. The significance level was set at alpha level of .05 prior to Bonferroni

296 correction. 
requested for the specific condition during both motor tasks $(\mathrm{M}=4.88 \sim 4.94, \mathrm{SD}=0.24 \sim 0.33)$.

301

302

303 mindfulness group interaction, $F(1,47)=6.12, p=.02, \eta^{2}=.12$ (see figure 1$)$. Further, we

Because the highest possible score is 5 , the scores indicated that participants followed the experimental design to use different types of self-talk. The descriptive data on motor task performance as well as a mindfulness group for the two self-talk conditions are presented in Table 2.

Insert table 2 about here

\section{Interaction effects of mindfulness and self-talk in standing long jump}

The 2 by 2 mixed design ANOVA showed no significant self-talk $\times$ mindfulness group interaction in standing long jump, $F(1,47)=0.001, p=.97$. However, the analysis revealed a main effect of self-talk on standing long jump, $F(1,47)=6.22, p=.02, \eta^{2}=.12$, indicating that participants performed better under instructional self-talk than unrelated self-talk. Further, there was no main effect of mindfulness group for standing long jump, $F(1,47)=0.01, p=.92$.

\section{Interaction effects of mindfulness and self-talk in line tracking}

Regarding line tracking, the 2 by 2 mixed design ANOVA showed a significant self-talk $\times$ utilized both independent t-test and paired t-test to compare this simple main effect of line 
316 tracking between mindfulness and self-talk. Under instructional self-talk, there was no difference

317 between high vs. low mindfulness participants in line tracking performance. However, under

318 unrelated self-talk, the independent t-test showed that high mindfulness performed better than

319 low mindfulness in line tracking, $t(47)=2.31, p=.01$, Cohen's $d=0.66$. Further, the paired t-test

320 showed that low mindfulness participants performed better under instructional self-talk than

321 unrelated self-talk, $t(22)=3.38, p=.00$, Cohen's $d=0.70$. However, for high mindfulness

322 participants, there was no difference in line tracking between instructional self-talk and unrelated

323 self-talk.

Insert figure 1 about here

325

Discussion

326

Theoretical Implications

327

In considering that mindfulness disposition might moderate the effects of self-talk on motor

328

task performance, the present study recruited participants with high vs. low mindfulness to

329 perform standing long jump and line tracking under instructional self-talk and unrelated self-talk

330

conditions. Results found several novel findings. First, an interaction effect was found between

331

mindfulness and self-talk in line tracking. Specifically, low mindfulness participants performed

332 poorer than high mindfulness participants in line tracking task under unrelated self-talk. Further

333 two main effects indicated that participants performed better in standing long jump and line 
334 tracking under instructional self-talk. However, high vs low mindfulness participants did not

335 differ in standing long jump and line tracking. The results provide several implications for

336 researchers.

337 First, our results confirmed that self-talk is beneficial to motor performance (e.g., Dickens,

338 Van Raalte, \& Hurlburt, 2018; Hardy, Comoutos, \& Hatzigeorgiadis, 2018; Weinberg, Miller, \&

339 Horn, 2012). Specifically, we found that all participants performed better both in standing long

340 jump and line tracking under the instructional self-talk condition. The effects of instructional

341 self-talk on motor performance may be due to performers focusing on relevant cues in the

342 environment and specific technical /mechanical aspects of the skills compared to those who did

343 not receive instructions (Abdoli et al., 2018; Boroujeni \& Shahbazi, 2011; Chang, et al., 2014;

344 Theodorakis et al., 2000).

345 The interaction of mindfulness disposition and self-talk in line tracking is very insightful for

346 researchers. To the best of our knowledge, this is the first study that includes mindfulness

347 disposition in examining the effects of self-talk on motor performance. Although past researchers

348 (e.g., Theodorakis et al., 2000; Zourbanos et al., 2013) suggested that attention might explain the

349 mixed results of the effects of the instructional self-talk on motor performance, no study has

350 examined the role of mindfulness disposition in self-talk-motor performance relationship. Our

351 study used mindfulness disposition as a moderator, and indirectly supported the moderating role 
352 of attention between instructional self-talk and motor performance relationship. Future study

353 may use a direct measure of attention, such as EEG and other neuro-physiological index, to

354 examine the moderation effect of attention on the self-talk- motor performance relationship (e.g.,

355 Martel, Dahne, \& Blankertz, 2014).

356 The interaction effect of mindfulness and self-talk on continuous motor task also outlines

357 the importance of instructional self-talk for low mindfulness participants. Especially, we found

358 low mindfulness participants performed better in line tracking only under instructional self-talk.

359 The results can be explained that instructional self-talk providing task-relevant cues or

360 information so they can perform well even with low ability to sustain their attention (Hardy,

361 2006; Hardy et al., 2001; Hatzigeorgiadis, Theodorakis, \& Zourbanos, 2004). However, the

362 results also found those participants with high mindfulness disposition did not differ under

363 instructional self-talk or unrelated self-talk in two motor tasks. This can be explained in that their

364 high ability to sustain attention from moment to moment helped them perform better with

365 different tasks either under instructional or unrelated self-talk conditions. Kee and Liu (2011)

366 suggested that high mindfulness participants performed better in different motor tasks because

367 they are kinesthetically aware and become responsive to motor tasks required. Thus, even under

368 unrelated self-talk condition, their performance was not impaired.

369 Further, under unrelated self-talk, high vs low mindfulness participants had no difference in 
370 standing long jump but had a significant difference in line tracking is worthy of discussion. This

371 unique result can be explained in that a discrete motor task such as standing long jump, is a rapid

372 and ballistic movement that requires a generalized motor program (GMP, Schmidt, 1975) to

373 perform the task. It requires a short time to sustain attention so it does not interfere low

374 mindfulness participants' performance under unrelated self-talk. In contrast, a continuous motor

375 task such as line tracking requires performers to sustain their attention to accomplish the task. If

376 during a continuous task a competing attention appeared (e.g., unrelated self-talk), it would

377 impair participants' performance, especially for those with low mindfulness disposition (Schmidt

$378 \&$ Lee, 2011, pp. 103-105). The unrelated self-talk can be a source of distraction for line tracking

379 that distracts low mindfulness performers' attention and makes errors. The central-resource

380 capacity theories of attention (McGill, 2002; pp. 143-145) proposed that there is motor

381 performance deficit when performing multiple activities at the same time for novel tasks or

382 unexperienced performers. Because there is limited capacity to receive and handle information, if

383 performers are beginners or unexperienced, they have difficulty receiving correct information to

384 react correctly. For our study, because low mindfulness participants tend to be less able to

385 sustain their attention, talking to themselves with unrelated words would distract their attention

386 during line tracking. In contrast, high mindfulness participants are characterized by an ability to

387 maintain attention from moment to moment. They were less distracted when speaking unrelated 
388 words. Therefore, further studies are needed to examine the performers' mindfulness disposition,

389 the role of attention allocation, type of self-talk, and motor task.

\section{Limitations and Future Suggestions}

391 Despite these significant and unique findings, several limitations should be considered when

392 interpreting the results of the present study. First, the operational and conceptual equating of

393 mindfulness disposition and attention ability may be a limitation, although related literature

394 suggests that high mindfulness disposition indicates high attention (Brown \& Ryan, 2002). Also,

395 empirical research supports that mindfulness induction can enhance attention through the

396 evidence of left dorsomedial prefrontal cortex activities (Doll et al., 2016), but we did not

397 measure left dorsomedial prefrontal cortex activities. Therefore, whether attention moderates the

398 effects of instructional self-talk on motor performance needs to be further examined. We suggest

399 future study may examine the effects of instructional self-talk on motor performance and

400 simultaneously measure EEG activities. By so doing, the role of attention in the instructional

401 self-talk - motor performance relationship can be further understood.

402 In addition, our study was conducted in a laboratory setting where participants were asked

403 to perform motor tasks under different self-talk conditions. Whether the results are applicable to

404 real athletic settings needs to be further examined. Specifically, a recent meta-analysis found

405 male expert athletes performed better on cognitive-motor tasks which require attentional abilities 
406 (Voss et al., 2010). Thus, future research needs to examine the effects of instructional self-talk

407 on motor tasks when participants are expert athletes or have sporting experience.

408 Further, because the duration of our self-talk intervention in the present study was adopted from

409 previous research (e.g., Chang et al., 2014; Boroujeni \& Shahbazi, 2011; Theodorakis et al.,

410 2000), whether a long-term intervention produces similar results needs to be further examined.

411 Specifically, some studies have conducted relatively long-term self-talk programs (4 to 10

412 weeks) and support their effectiveness for sports performance (Hamilton et al., 2007;

413 Hatzigeorgiadis, Galanis, Zourbanos, \& Theodorakis, 2013; Zetou et al., 2012). Moreover, we

414 suggest future studies examine the effects of instructional self-talk in varied settings. Martin,

415 Vause, and Schwartzman (2005) indicated that most self-talk research is conducted in laboratory

416 settings. Therefore, future studies may examine how instructional self-talk in competitive sports

417 settings, academic settings, fitness centers, military, sports injury, and rehabilitation. Moreover,

418 we found no difference in discrete and continuous tasks for high vs low mindfulness participants.

419 The true reasons for this finding are unknown. We suggest future studies to examine how

420 mindfulness disposition interacts with external stimuli on different motor tasks under different

421 self-talk conditions.

\section{Applications}

423 Practitioners, such as PE teachers, coaches, and sport psychology consultants can apply our 
424 results in various settings. To facilitate students or athletes' motor learning and control

425 practitioners should provide positive and task-relevant information to performers (Edwards,

426 2011; pp. 362-401). Specifically, practitioners should provide both the general and specific

427 aspects of the skills so performers can understand the key components of the skills and direct

428 their attention to those parts when performing the motor skills. Also, practitioners should avoid

429 giving unrelated information to prevent distracting attention. In addition, because there is limited

430 information processing ability for beginners or unexperienced students, it is suggested that PE

431 teachers, coaches, and sports psychology consultants should give each key instruction each time

432 when learning a new skill. Also, the instructors should give precise information about the skill

433 requirements and avoid jargon (Edwards, 2011, pp. 366-367). For those students with high

434 mindfulness, it is suggested that PE teachers and coaches could provide a different level of

435 assignments and materials, and foster independent learning (Laine \& Tirri, 2015).

436

437 Conclusion

438 Self-talk is a very useful psychological skill to enhance performers' motor skill learning and

439 control. Our study provides evidence that instructional self-talk is beneficial to both discrete and

440 continuous motor skills. Additionally, we found participants' mindfulness disposition interacts

441 with self-talk on the motor task. Future research may extend our research by directly examining 
442 performers' mindfulness disposition, the role of attention allocation, type of self-talk, and motor

443 task to unveil the interweaving relationships of instructional self-talk, attention, and motor

444 performance. Moreover, when applying instructional self-talk with athletes and PE students,

445 practitioners should provide positive and task-related instruction in order to facilitate performers'

446 motor task performance.

447

448

\section{References}

449 Abdoli, B., Hardy, J., Riyahi, J. F., \& Farsi, A. (2018). A closer look at how self-talk influences

450 skilled basketball performance. The Sport Psychologist, 32, 9-15. doi:10.1123/tsp.2016-

$451 \quad 0162$

452 Anderson, J. R. (2004). Cognitive Psychology and Its Implications (6th ed.). Worth

453 Publishers. ISBN 978-0-7167-0110-1.

454 Boroujeni, S. T., \& Shahbazi, M. (2011). The effect of instructional and motivational self-talk on

455 performance of basketball's motor skill. Procedia-Social and Behavioral Sciences, 15,

456 3113-3117. DOI:10.1016/j.sbspro.2011.11.282. 
457 Brown, K. W., \& Ryan, R. M. (2003). The benefits of being present: Mindfulness and its role in

458 psychological well-being. Journal of Personality and Social Psychology, 84, 822-848.

459

PubMed DOI:10.1037/0022-3514.84.4.822

460 Chang, Y. K., Ho, L. A., Lu, F. J. H., Ou, C. C., Song, T. F., \& Gill, D. (2014). Self-talk and

461 softball performances: The role of self-talk nature, motor task characteristics, and self-

462 efficacy in novice softball players. Psychology of Sport and Exercise, 15,135-149. DOI:

$463 \quad$ 10.1016/j.psychsport.2013.10.004

464 Dickens, Y.L., Van Raalte, J., \& Hurlburt, R.T. (2018). On investigating self-talk: A descriptive

465 experience sampling study of inner experience during golf performance. The Sport

466 Psychologist, 32(1) DOI:10.1123/ tsp.2016-0073

467 Djikic, M., Langer, E. J., \& Stapleton, S. F. (2008). Reducing stereotyping through mindfulness:

468 Effects on automatic stereotype-activated behaviors. Journal of Adult Development, 15(2),

469 106-111. DOI:10.1007/s10804-008-9040-0

470 Doll, A., Holzel, B. K., Bratek S.M., Boucard, C. C., Xie, X., Wohlschläger, A. A., \& Sorg, C.

471 (2016). Mindful attention to breath regulates emotions via increased amygdala-prefrontal 
472 cortex connectivity. NeuroImage, 134 (1), 305-313. DOI:

$473 \quad$ 10.1016/j.neuroimage.2016.03.041

474 Edwards, W. H. (2011). Motor Learning and Control: From Theory to Practice. Belmont CA:

$475 \quad$ Wadsworth, Cengage Learning.

476 Edwards, C., Tod, D., \& McGuigan, M. (2008). Self-talk influences vertical jump performance

477 and kinematics in male rugby union players. Journal of Sports Sciences, 26, 1459-1465.

$478 \quad$ DOI:10.1080/02640410802287071.

479 Fleishman, E. A. (1954). Dimensional analysis of psychomotor abilities. Journal of Experimental

480 Psychology, 48(6), 437-454. DOI:10.1037/h0058244

481 Gould, D., Murphy, S., Tammen, V., \& May, J. (1991). An evaluation of U.S. Olympic sport

482 psychology consultant effectiveness. The Sport Psychologist, 5, 111-127. DOI:

$483 \quad 10.1123 /$ tsp.5.2.111

484 Hamilton, R. A., Scott, D., \& MacDougall, M. P. (2007). Assessing the effectiveness of self- talk 485 interventions on endurance performance. Journal of Applied Sport Psychology, 19, $226-239$.

486 DOI: $10.1080 / 10413200701230613$ 
487 Hardy, J. (2006). Speaking clearly: a critical review of the self-talk literature. Psychology of Sport and Exercise, 7, 81-97. DOI:10.1016/ j.psychsport.2005.04.002.

489

490

491

492

493

494

495

496

497

498

499

500

501

502

503

504

Hardy, J., Begley, K., \& Blanchfield, A. W. (2015). It's good but it's not right: Instructional selftalk and skilled performance. Journal of Applied Sport Psychology, 27(2), 132-139. DOI:

$10.1080 / 10413200.2014 .959624$

Hardy, J., Comoutos, N., \& Hatzigeorgiadis, A. (2018). Reflections on the maturing research literature of self-talk in sport: Contextualizing the Special Issue. The Sport Psychologist, 32(1), 1-8. DOI: 10.1123/tsp.2017-0141

Hardy, J., Gammage, K., \& Hall, C. R. (2001). A description of athlete self-talk. The Sport Psychologist, 15, 306-318. DOI: 10.1123/tsp.15.3.306

Hatzigeorgiadis, A., Theodorakis, Y., \& Zourbanos, N. (2004). Self-talk in the swimming pool: The effects of ST on thought content and performance on water-polo tasks. Journal of Applied Sport Psychology, 16, 138-150. DOI: 10.1080/10413200490437886

Hatzigeorgiadis, A., Zourbanos, N., Galanis, E., \& Theodorakis, Y. (2011). Self-talk and sports performance: A meta-analysis. Perspectives on Psychological Science, 6, 348-356.

DOI:/10.1177/1745691611413136.

Hatzigeorgiadis, A., Zourbanos, N., Mpoumpaki, N., \& Theodorakis, N. (2009). Mechanisms underlying the self-talk-performance relationship: The effects of motivational self-talk on 
505

506

507 Hatzigeorgiadis, A., Galanis, V., Zourbanos, N., \& Theodorakis, Y. (2014). A self-talk

508

509

510

511

512

513 Kee, Y. H., Chatzisarantis, N. L. D., Kong, P. W., Chow, J. Y., \& Chen, L. H. (2012).

514

515

516

517 Kolovelonis, A., Goudas, M., \& Dermitzaki, I. (2011). The effects of instructional and

518

519

520 Landin, D. K. (1994). The role of verbal cues in skill learning. Quest, 46, 299-313. 
521 Laine, S., \& Tirri, K. (2015). How Finnish elementary school teachers meet the needs of their

522 gifted students. High Ability Studies, 27 (2), 149-164, DOI:

$523 \quad 10.1080 / 13598139.2015 .1108185$.

524 Lewin, K. (1953). A dynamic theory of personality. New York: McGraw-Hill.

525 Martel, A., Dahne, S., \& Blankertz, B. (2014). EEG predictors of covert vigilant attention.

526 Journal of Neural Engineering, 11, 035009, (11pp) DOI:10.1088/1741-2560/11/3/035009

527 Martin, G. L., Vause, T., \& Schwartzman, L. (2005). Experimental studies of psychological

528 interventions with athletes in competitions: Why so few? Behavior Modification, 29, 616-

529 641. DOI:10.1177/0145445503259394

530 McGill, R. A. (2003). Motor Learning and Control: Concepts and Applications. McGraw

531 Hill.

532

533

534

535 Nideffer, R. M. (1993). Concentration and attention control training. In J. Williams (Ed.),

Mills, N., \& Allen, J. (2000). Mindfulness of movement as a coping strategy in multiple sclerosis: A pilot study. General Hospital Psychiatry, 22(6), 425-431. DOI:10.1016/ S0163-8343(00)00100-6 Applied sport psychology (pp. 243-262). Palo Alto: Mayfield. 
537 Schmidt, R. A. (1975). A schema theory of discrete motor skill learning. Psychological Review,

538 82, 225-260. DOI: $10.1037 / \mathrm{h} 0076770$

539 Schmidt, R. A., \& Lee, T. D. (2011). Motor control and learning: A behavioral emphasis (5th

540 ed.). Champaign, IL, US: Human Kinetics.

541 Schmid, G., Sauter, C., Stepansky, R., Lobentanz, I. S., \& Zeitlhofer, J. (2005). No influence on

542 selected parameters of human visual perception of $1970 \mathrm{MHz}$ UMTS-like

543 exposure. Bioelectromagnetics, 26, 243-250.DOI: 10.1002/ bem.20076

544 Thomas, J. R., Nelson, J.K., \& Silverman, S.J. (2015). Research Method in Physical Activity.

$545 \quad$ Human Kinetics. Champaign, IL.

546 Theodorakis, Y., Weinberg, R., Natsis, P., Douma, I., \& Kazakas, P. (2000). The effects of

547 motivational and instructional self-talk on improving motor performance. The Sport

548 Psychologist, 14, 253-271.DOI: 10.1123/tsp.14.3.253

549 Tod, D. A., Hardy, J., \& Oliver, E. (2011). Effects of self-talk: A systematic review. Journal of 550 Sport and Exercise Psychology, 33, 666-687. DOI: 10.1123/jsep.33.5.666

551 Voss, M. W., Kramer, A. F., Basak, C., Prakash, R. S., \& Roberts, B. (2010). Are Expert 552 Athletes 'Expert' in the Cognitive Laboratory? A Meta-Analytic Review of Cognition and Sport Expertise, Applied Cognitive Psychology, 24, 812-826. DOI: 10.1002/acp.1588 
554 Westphal, W., \& Porter, J. M. (2013). Increasing the distance of an external focus of attention

555

556

557

558

559

560

561

562

563

564

565

566

567

568

569

has limited effects on standing long jump performance. International Journal of Exercise

Science, 6(4), 300-309.

Weinberg, R. \& Gould, D. (2015). Foundations of Sport and Exercise Psychology (6th Edition).

United States of America: Courier Companies, Inc.

Weinberg, R., Miller, A., \& Horn, T. (2012). The influence of a self-talk intervention on collegiate cross-country runners. International Journal of Sport and Exercise Psychology, 10, 123-134. DOI;10.1080/1612197X.2012.645135.

Williams, J. M. \& Krane, V. (2015). Sport psychology: Past, present, future. In J. M. Williams \& V. Krane (Eds.), Applied sport psychology: Personal growth to peak performance ( $7^{\text {th }}$ ed., pp. 1-15). New York: McGraw-Hill.

Zetou, E., Vernadakis, N., Bebetsos, E., \& Makraki, E. (2012). The effect of self-talk in acquisition and learning of the volleyball service skill and self-efficacy improvement. Journal of Human Sport and Exercise, 7 (4), 794-805.

DOI:10.4100/jhse.2012.74.07

PeerJ reviewing PDF | (2018:10:32358:3:1:NEW 24 Apr 2019) 
570 Zetou, E., Nikolaos, V., \& Evaggelos, B. (2014). The effect of instructional self-talk on

571 performance and learning the backstroke of young swimmers and on the perceived

572 functions of it. Journal of Physical Education and Sport, 14(1), 27-35.

$573 \quad$ DOI:10.7752/jpes.2014.01005

574 Zhang, J., Xu, R., Wang, B., \& Wang, J. (2016). Effects of mindfulness-based therapy for

575 patients with breast cancer: A systematic review and meta-analysis. Complementary

576 Therapies in Medicine, 26, 1-10. DOI: 10.1016/ j.ctim.2016.02.012

577 Zourbanos, N., Hatzigeorgiadis, A., Bardas, D., \& Theodorakis, Y. (2013). The effects of self-

578 talk on dominant and nondominant arm performance on a handball task in primary physical

579 education students. The Sport Psychologist, 27, 171-176. DOI: 10.1123/tsp.27.2.171 
Figure 1 (on next page)

Interaction of mindfulness and two types of self-talk on line tracking

This figure illustrates how high vs low mindfulness disposition performed differently on line tracking under different self-talks 
Table $\mathbf{1}$ (on next page)

Demographic characteristics of participants

Demographic Characteristics of Participants $(\mathrm{N}=49)$. 
1 Table 1. Demographic Characteristics of Participants $(\mathrm{N}=49)$.

2

\begin{tabular}{llllll}
\hline Measures & Male & Female & $\begin{array}{l}\text { High } \\
\text { mindful } \\
\text { group }\end{array}$ & $\begin{array}{l}\text { Low } \\
\text { mindful } \\
\text { group }\end{array}$ & Total \\
\hline$n$ & 26 & 23 & 26 & 23 & 49 \\
Age & $19.08 \pm 1.32$ & $18.83 \pm 0.72$ & $18.69 \pm 0.62$ & $19.26 \pm 1.39$ & $18.96 \pm 1.08$ \\
Height & $174.58 \pm 6.00$ & $160.78 \pm 5.41$ & $168.69 \pm 10$. & $167.43 \pm 6.5$ & $168.10 \pm 8.96$ \\
Weight & $69.31 \pm 14.20$ & $51.43 \pm 6.76$ & $61.62 \pm 17.0$ & $60.13 \pm 11.0$ & $60.92 \pm 14.39$ \\
BMI & $22.78 \pm 4.74$ & $19.87 \pm 2.15$ & $21.38 \pm 4.26$ & $21.44 \pm 3.77$ & $21.41 \pm 4.00$ \\
\hline
\end{tabular}

Note. $\mathrm{BMI}=$ body mass index.

3 


\section{Table 2 (on next page)}

Descriptive Statistics for All Participants on Two Motor Tasks Under Different Self-Talks

This table illustrates all the statistics of all participants' performance on two types of motor tasks under different self-talk 
1 Table 2. Descriptive Statistics for All Participants on Two Motor Tasks Under Different Self-

2 Talks.

\begin{tabular}{lll}
\hline Variable & Instructional & Unrelated \\
\hline $\begin{array}{l}\text { Standing Long Jump } \\
(\mathrm{N}=49)\end{array}$ & $171.82 \pm 40.21$ & $166.88 \pm 37.15$ \\
\hline low mindfulness $(\mathrm{n}=23)$ & $171.17 \pm 38.20$ & $166.30 \pm 35.07$ \\
high mindfulness & $172.38 \pm 42.65$ & $167.38 \pm 39.59$ \\
$(\mathrm{n}=26)$ & & \\
\hline Line Tracking Test & $13.18 \pm 7.16$ & $16.36 \pm 10.31$ \\
$(\mathrm{~N}=49)$ & & $19.83 \pm 12.05$ \\
\hline low mindfulness $(\mathrm{n}=23)$ & $14.39 \pm 6.95$ & $13.29 \pm 7.47$ \\
high mindfulness & $12.12 \pm 7.31$ & \\
$(\mathrm{n}=26)$ & & \\
\hline
\end{tabular}

3

4

5 